\section{Welcome addition to multivariate analysis}

Discrete Multivariate Analysis: Theory and Practice. By Yvonne M. M. Bishop, Stephen E. Fienberg and Paul W. Holland. Pp. $x+557$. (MIT Press: Cambridge, Massachusetts, and London, March 1975.) \$27.50.

Discrete data, in which each variable takes one of a limited number of possible forms, arise frequently in many fields. Multivariate analysis of such data is required when the joint distribution of several variables is to be studied. The present book gives an account of statistical methods for these problems. It concentrates largely, although not entirely, on an approach in which the $\log$ frequencies in cells of a multidimensional contingency table are represented in some simple form, the so-called log linear model. To an appreciable extent the book is based on the authors' earlier important con. tributions to this topic.

The discussion is lucid and very leisurely, excellently illustrated with applications drawn from a wide variety of fields. A good part of the book can be understood without very specialised statistical knowledge. It is a most welcome contribution to an interesting and lively subject.

After an introductory chapter, structural models for multidimensional discrete data are described, and then maximum likelihood methods of estimation are developed; this is followed by a consideration of the methods used for testing degree of fit. Methods for incomplete data (missing cells) are discussed at length. There is an interesting chapter on estimating the size of a closed population, in which the usual methods for the analysis of a multiple capture-recapture census are modified to take account of possible dependencies between the samples on successive occasions. Further chapters include discussions on Markov models for assessing change and on the special problems of 'square' tables. The book ends with two chapters on approaches other than that of the log linear model and chapters on pseudo-Bayes estimates (that is, on smoothing tables), on sampling models and on mathematical aspects.

The scope of the book is rather less than might have been expected from its title. For instance, there is no discussion of latent structure analysis or of the difficult problems of analysing multivariate discrete data in connection with numerical taxonomy.

I have one general criticism, which, though to some extent a matter of taste, seems to me important. The authors barely mention the distinction between response variables (dependent variables) and explanatory variables (independent variables). Yet in quite a number of their examples the point at issue seems to concern how variation in one of the variables is explained by the other variables, which is a univariate and not a multivariate problem. Even if the detailed analysis is little affected, incisive explanation of conclusions is much easier for univariate problems. At a more technical level the authors have ignored the fact that in somebut not, of course, all-of their problems conditioning on ancillary statistics is possible.

D. R. Cox

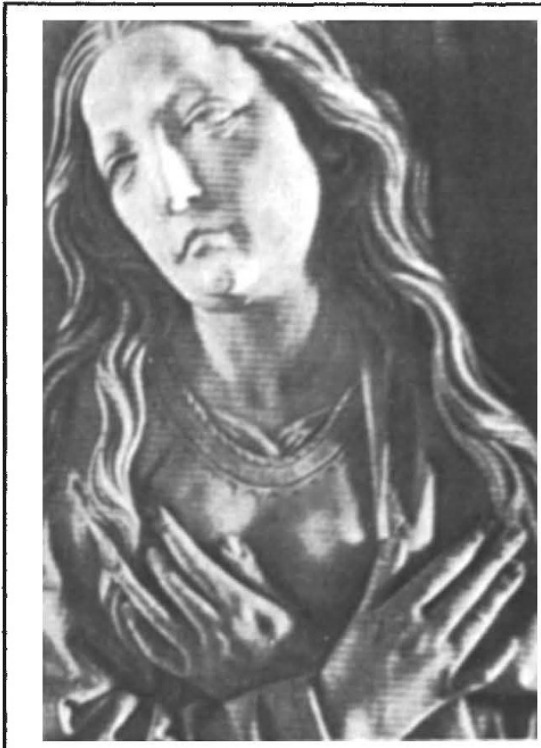

The Virgin Mary, hands crossed in the gesture of humble submission. A wood carving from the Marienalter of Herrgottskirche in Creglingen, Germany. Photograph taken from Silent Language, by Macdonald Critchley. Pp. vi + 231. (Butterworth: London, April 1975.) $£ 9.50$.

\section{Fundamentals}

Fundamentals of Mathematics. Vol. 1: Foundations of Mathematics/The Real Number System and Algebra. Pp. x+549. Vol. 2: Geometry. Pp. xi +685 . Vol. 3: Analysis. Pp. xiii +541 . Edited by $\mathrm{H}$. Behnke, F. Bachmann, K. Fladt and W. Suss. (MIT Press: Cambridge, Massachusetts, and London, 1974.) \$15.95 each volume; $\$ 40.00$ the set.

THIS work stands alone; it is a unique survey of the whole field of pure mathematics, with emphasis on the basic ideas and their interrelationships. For its origin, we must go back over 20 years. In 1954 the International Commission for Mathematical Instruction, at a meeting in Paris, chose the scientific foundations of instruction in mathematics as one topic for the 1958 International Congress in Edinburgh. As a result, the German section of the Commission began Volume I of this work. During its subsequent development authors from various European countries contributed, and it became clear that the material would be important not only for teachers and lecturers but also for research mathematicians. The original publication was in German, and the volumes now reviewed consist of a translation based on the second German edition of 1962 ; it incorporates various improvements and exercises designed to bring the account up to date.

Each chapter has been written by two authors, one a university research mathematician, the other "a teacher of long experience in the educational system". As a result, over 80 authors have contributed.

Volume I consists of a part on the foundations of mathematics and a part on arithmetic and algebra. Volume II deals with geometry, and consists of 16 chapters covering both the foundations and the analytic treatment of this aspect of mathematics. Worthy of special mention are the chapters on geometrical constructions and on polygons and polyhedra. Volume III is concerned with analysis and includes 14 chapters.

No mathematical specialist can consult the work without becoming acutely aware of the tremendous breadth of mathematical thought and discovery. The student, contemplating research in some area of the subject, would do well to read, in these volumes, of the relationship between that particular area and the subject at large; for it is a distinctive feature of the work that it emphasises the connections between different branches of mathematics.

Many people, including a lot of mathematicians, find it difficult to see perspective in mathematics. To such, the last chapter, on the changing structure of modern mathematics, is to be commended. Here is a very clear account, carefully traced, of what has been happening in recent decades. It is all summed up in the expression "axiomatic way of thinking". There is reference, of course, to the Bourbaki movement in France, and to the development of the axiomatic method in geometry. Then comes the change in algebra, namely the breakthrough of axiomatic algebra (covered particularly well by E. Artin and Emmy Noether). Finally, the great achievements of the axiomatic method in analysis are traced, although these considerations are tinged with an element of doubt as to whether the axiomatic change in analysis will ever be as complete as that in algebra.

No serious mathematician can afford to neglect these volumes. To read them is an experience; and, at the end, the reader will be more aware of the abiding serenity of his subject.

L. S. Goddard 